\title{
Popularization of exercise rehabilitation in the information age
}

\section{Khae-Hawn Kim}

As we get closer to the middle of this year, it is natural to question what lies ahead in the year 2015. Recently, the literature in the Journal of Exercise Rehabilitation (JER) has focused on the future of exercise rehabilitation based on three potential scenarios arising from the ongoing information revolution that marginalizes some communities and creates new opportunities for others. The first scenario is that of rehabilitation ruled by the "general public," where exercise is largely administered based on recommendations by consumer agglomerations. The second possibility is that of rehabilitation in which strong associations use large amounts of data obtained from the popularly used exercises to fortify their data. In the third scenario, the central associations are fundamentally weak, with markets - and the society that dominates them - providing almost all exercises or activities for the disabled and children and for recreational and psychological rehabilitation. Each of these scenarios is an extrapolation of a current trend. While all of them could be beneficial in some respects, they also have features, which if left scientifically and medically unchecked, could lead to disappointing outcomes. The Association of Korean Exercise Rehabilitation should, at this time, be implementing policies aimed at guiding trends like generalization, increasing the amount of available data, and grouping people into narrow branches often based on their relationship to the region. The goal should be to take advantage of these trends' potential benefits, while ensuring that they do not undermine other critical aspects of exercise rehabilitation. For example, although exercise has the potential to create new treatment and preventative opportunities for potential patients, it cannot help cure actual diseases. Likewise, while the use of large amounts of general public data has a substantial problem-solving potential, important questions regarding the ownership, control, interpretation, and regulation of the use of this data or information still remain. Finally, while the most efficient way to allocate exercise rehabilitation resources in most cases is to do so based on individual choices within each region, regional branches do not develop sufficient remedial exercise programs. Indeed, there are some exercise methods that the private and regional sectors are simply unable to provide. Obviously, all of this calls for an extensive investigation. Exploring potential future scenarios, as done by the JER, is an important step in the right direction.

Editor-in-Chief Department of Urology, Gachon University Gil Medical Center, 21 Namdong-daero 774beon-gil, Namdong-gu, Incheon 405-760, Korea E-mail: kimcho99@gilhospital.com

This is an Open Access article distributed under the terms of the Creative Commons Attribution Non-Commercial License (http://creativecommons.org/licenses/by-nc/3.0/) which permits unrestricted non-commercial use, distribution, and reproduction in any medium, provided the original work is properly cited. 